\title{
GGsTOP increases migration of human periodontal ligament cells in vitro via reactive oxygen species pathway
}

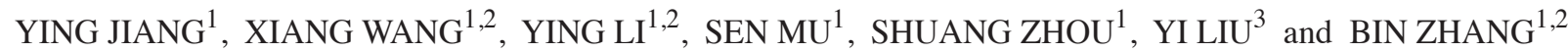 \\ ${ }^{1}$ Institute of Hard Tissue Development and Regeneration, The Second Affiliated Hospital of Harbin Medical University; \\ ${ }^{2}$ Heilongjiang Academy of Medical Sciences, Harbin, Heilongjiang 150001; ${ }^{3}$ College of Stomatology, \\ Tongji University, Shanghai 200072, P.R. China
}

Received May 20, 2015; Accepted March 7, 2016

DOI: $10.3892 / \mathrm{mmr} .2016 .5038$

\begin{abstract}
GGsTOP is a novel and selective inhibitor of gamma-glutamyl transferase (GGT), a cell-surface enzyme that has a key role in glutathione homeostasis and the maintenance of cellular reactive oxygen species (ROS). ROS are essential for wound healing. However, little is known about the molecular mechanisms underlying the inhibition of GGT by GGsTOP in human periodontal ligament cells (hPLCs). The present study assessed GGT expression in mouse periodontal ligament tissues, GGT activity in hPLCs, and the potential physiological effect of GGsTOP on hPLC migration. Immunohistochemical analysis confirmed that GGT was widely expressed in mouse periodontal ligament tissue. Treatment with GGsTOP was associated with greater proliferation and migration of hPLCs, and higher levels of cellular ROS compared with untreated hPLCs. However, the increase in intracellular ROS was attenuated in hPLCs co-cultured with the anti-oxidant $N$-acetylcysteine (NAC), a precursor of glutathione. The higher ROS levels associated with GGSTOP treatment were in parallel with increases in the levels of type I collagen and alpha smooth muscle actin, which was inhibited in hPLCs co-cultured with NAC. Thus, GGsTOP may promote hPLC migration and participate in the maintenance of the periodontal ligament apparatus via the ROS pathway.
\end{abstract}

\section{Introduction}

Periodontitis is a highly prevalent inflammatory disease that causes the destruction of connective tissue, resorption of surrounding bone tissue and eventually tooth loss. Therapy

Correspondence to: Professor Bin Zhang, Institute of Hard Tissue Development and Regeneration, The Second Affiliated Hospital of Harbin Medical University, 246 Xue Fu Road, Harbin, Heilongjiang 150001, P.R. China

E-mail: doc_zhangb@126.com

Key words: reactive oxygen species, cell migration, periodontal ligament, gamma-glutamyl transferase, alpha-smooth muscle actin for periodontitis consists of controlling inflammation and promoting healing. The remaining healthy periodontal ligament (PDL) has an essential role by retaining its potential for regeneration to a certain extent throughout adulthood. The PDL is composed of several different cell populations that are characterized by their ability to remodel and renew periodontium (1). In particular, PDL fibroblasts are the key cells involved in these processes; during periodontal wound healing, fibroblasts are activated, migrate to the wound site and repair damaged tissue by remodeling the extracellular matrix (2).

From a biological perspective, the wound healing process involves a complexity of cellular biochemical events. It is necessary to maintain a delicate balance between the positive and deleterious effects of reactive oxygen species (ROS) during wound healing and successful tissue regeneration. ROS are chemically reactive molecules containing oxygen. High ROS levels were originally considered to be closely linked to aging and various degenerative diseases, including stroke and cardiovascular disease. However, a number of studies have shown that ROS have a physiological role in several non-phagocytic cells. It is now widely recognized that at low concentrations, ROS act as ubiquitous intracellular messengers that support wound healing (3). Increased ROS generation has been associated with the mitogenic stimulation of growth factors (4).

Glutathione is a non-protein thiol found in mammalian cells, and is essential for maintaining intracellular ROS balance and the thiol status of proteins (5).

Gamma-glutamyl transferase (GGT) is the only cell-surface enzyme that catalyzes the initial step of extracellular glutathione degradation, thereby providing additional cysteine, which is the rate-limiting substrate for intracellular de novo glutathione synthesis (6). Therefore, catabolism of glutathione by GGT affects intracellular ROS levels and cysteine homoeostasis (7). GGsTop is a novel phosphonate-based, irreversible inhibitor of GGT, and exhibits a much higher inhibitory activity than the traditional GGT inhibitor, acivicin. Furthermore, GGsTOP inhibits only GGT, and does not have any influence on glutamine amidotransferases (8).

Based on the above evidence, the present study hypothesized that GGT may be produced in the PDL. In addition, the inhibition of GGT activity by GGsTOP may affect intracellular ROS levels, and thereby influence the remodeling and renewal 
of human periodontal ligament cells (hPLCs) to induce the fiber synthesis necessary to repair PDL tissues. Hence, the objective of the present study was to investigate the levels of GGT in PDL tissues, and to evaluate the effects of the inhibition of GGT activity on hPLCs.

\section{Materials and methods}

Reagents. The reagents dichlorodihydrofluorescein diacetate (DCFH-DA), 4',6-diamidino-2-phenylindole dihydrochloridedimethyl sulfoxide (DAPI), $N$-acetyl-L-cysteine (NAC) were purchased from Sigma-Aldrich (St. Louis, MO, USA). GGsTop (Wako Pure Chemical Industries, Osaka, Japan) is freely soluble in distilled water; therefore, a $10-\mathrm{mM}$ stock solution was prepared, stored at $-20^{\circ} \mathrm{C}$, and for subsequent experiments diluted to different concentrations with Dulbecco's modified Eagle's medium (DMEM; Gibco; Thermo Fisher Scientific, Inc., Waltham, MA, USA).

Isolation and cell culture of hPLCs. Human tissue sample collection was approved by the ethics committee of the Second Affiliated Hospital of Harbin Medical University (Harbin, China), and informed consent was obtained from all patients or their advisers prior to teeth extraction, according to the ethics committee guidelines. A total of 8 healthy premolar teeth were collected from four 12-16 adolescents (two males and two females) who had extracted teeth for orthodontic reasons with no evidence of caries, periodontitis or gingivitis. The periodontal ligament tissues were scraped from the middle one-third of the tooth roots, minced and then placed in $25-\mathrm{cm}^{2}$ culture flasks containing DMEM with $10 \%(\mathrm{v} / \mathrm{v})$ fetal bovine serum (GE Healthcare Life Sciences, Logan, UT, USA), $100 \mathrm{U} / \mathrm{ml}$ penicillin and $100 \mu \mathrm{g} / \mathrm{ml}$ streptomycin (Gibco; Thermo Fisher Scientific, Inc.). The tissues were incubated at $37^{\circ} \mathrm{C}$ in $5 \% \mathrm{CO}_{2}$ and $100 \%$ relative humidity. Cells were passaged until $80 \%$ confluent, and cells from the third to fifth passages were used for the experiment.

Immunohistochemistry. Male C57BL/6 mice $(\mathrm{n}=4$; age, four weeks; weight, 20-30 g) were purchased from the experimental animal center of the Second Affiliated Hospital of Harbin Medical University. The animal experiments were approved by the ethics committee of the Second Affiliated Hospital of Harbin Medical University. The mice were anesthetized with $10 \%$ chloral hydrate $(250 \mathrm{mg} / \mathrm{kg}$; Wuhan Hechang Chemical Co., Ltd., Wuhan, China) by intraperitoneal injection and perfused transcardially with $4 \%$ paraformaldehyde (Tianjin Guangfu Chemical Co., Ltd., Tianjin, China) in phosphate-buffered saline (Beijing Solarbio Science \& Technology Co., Ltd., Beijing, China). The mandibles were removed and immersed in $4 \%$ paraformaldehyde at $4^{\circ} \mathrm{C}$ for $48 \mathrm{~h}$. The tissues were subsequently decalcified and embedded in paraffin (Shanghai Hushi Laboratorial Equipment Co., Ltd, Shanghai, China). Five-micron-thick sections were analyzed by immunohistochemistry using a mouse monoclonal antibody against gamma-glutamyl transferase (1:400 dilution; cat. no. ab55138; Abcam, Cambridge, MA, USA). The sections were then incubated with a biotinylated secondary antibody using a Histostain-Plus kit (ZSGB-BIO, Beijing, China). The color reaction was finally developed using 3,3'-diaminoben- zidine (Nichirei, Tokyo, Japan). The sections were observed using a light microscope (Eclipse 80i; Nikon, Tokyo, Japan).

Determination of GGT activity. Determination of GGT activity was performed as described by Huseby and Srömme (9) using $22.5 \mathrm{mmol} / 1 \mathrm{\gamma}$-glutamyl-p-nitroanilide (Sigma-Aldrich) as the donor and $123 \mathrm{mmol} / \mathrm{l}$ glycylglycine (Sigma-Aldrich) as the glutamate acceptor, with a volume fraction of 1:11. Confluent cell monolayers of human hepatoma HepG2 cells (American Type Culture Collection, Manassas, VA, USA) and hPLCs were pre-treated with GGsTOP for $2 \mathrm{~h}$ followed by further treatment with hypotonic lysis buffer $(10 \mathrm{mM}$ Tris- $\mathrm{HCl}, \mathrm{pH}$ 7.8), and ground using a glass Dounce homogenizer (3432N75; Thomas Scientific, Swedesboro, NJ, USA) (30 strokes; $4^{\circ} \mathrm{C}$ ). The cells were collected separately and amounts of p-nitroaniline formed were measured by spectrophotometry at $405 \mathrm{~nm}$ using a $722 \mathrm{~S}$ Visible spectrophotometer (Shanghai Inesa Scientific Instrument, Shanghai, China). The units of enzyme activity were calculated using a molar extinction coefficient of 9.9 for p-nitroanilide. One unit of GGT activity was defined as the amount of enzyme required to release $1 \mu \mathrm{mol}$ of substrate transformed $/ \mathrm{ml} / \mathrm{min}$. The results were expressed as $\mathrm{U} / \mathrm{g}$ cell protein. Protein content was measured using the bicinchoninic acid (BCA) method (Beyotime Institute of Biotechnology, Haimen, China).

Cell proliferation. Cell viability was measured in hPLCs using the 3-(4,5-dimethylthiazol-2-yl)-2,5-diphenyltetrazolium bromide (MTT) assay. In brief, hPLCs $\left(2.5 \times 10^{3}\right.$ cells/well) were seeded into 96-well plates and allowed to attach and grow for $24 \mathrm{~h}$. The cells were starved overnight and then incubated with $0,5,10,20$ or $50 \mu \mathrm{M}$ GGsTOP for $1,3,5$ and 7 days. The medium was replaced every other day. Then, $20 \mu \mathrm{l}$ MTT (Sigma-Aldrich) was added to each well at the indicated time point and the cells were incubated for $4 \mathrm{~h}$. The medium was subsequently removed and $150 \mu \mathrm{l}$ dimethyl sulfoxide (Sigma-Aldrich) was added to each well for $10 \mathrm{~min}$. The absorbance was determined at $490 \mathrm{~nm}$ with a microplate reader (Model 550; Bio-Rad Laboratories, Inc., Hercules, CA, USA).

Generation of ROS. Cellular levels of ROS were measured by means of the previously described semi-quantitative DCFH-DA fluorescence technique, which can be used to track changes in the ROS concentration over time (10). The hPLCs were seeded into six-well plates at a density of $7 \times 10^{5}$ cells/well and starved overnight. The cells were then pre-incubated with the fluorescent dye DCFH-DA $(10 \mathrm{mM} / 1$ in DMEM) for $20 \mathrm{~min}$ at $37^{\circ} \mathrm{C}$ and $5 \% \mathrm{CO}_{2}$ in the dark. After incubation with the dye, the cells were rinsed twice in phosphate-buffered saline prior to treatment with GGsTOP $(10 \mu \mathrm{M})$ or GGsTOP $(10 \mu \mathrm{M})+\mathrm{NAC}(3 \mathrm{mM})$; cells without any treatment were used as controls. Cells from all groups were subjected to fluorescence microscopy at $0,5,10,15,30,60,120$ and $180 \mathrm{~min}$. Six random fields at the center of each well (border of $4 \times 4 \mathrm{~mm}$ ) were analyzed at excitation and emission wavelengths of 485 and $525 \mathrm{~nm}$ using a fluorescence microscope (Eclipse Ti-S, Nikon). The results were analyzed by NIS-Elements Basic Research software 4.10 (Nikon). Values are expressed as the percentage change in fluorescence at the pixel level compared with that at time 0 (baseline). 
A
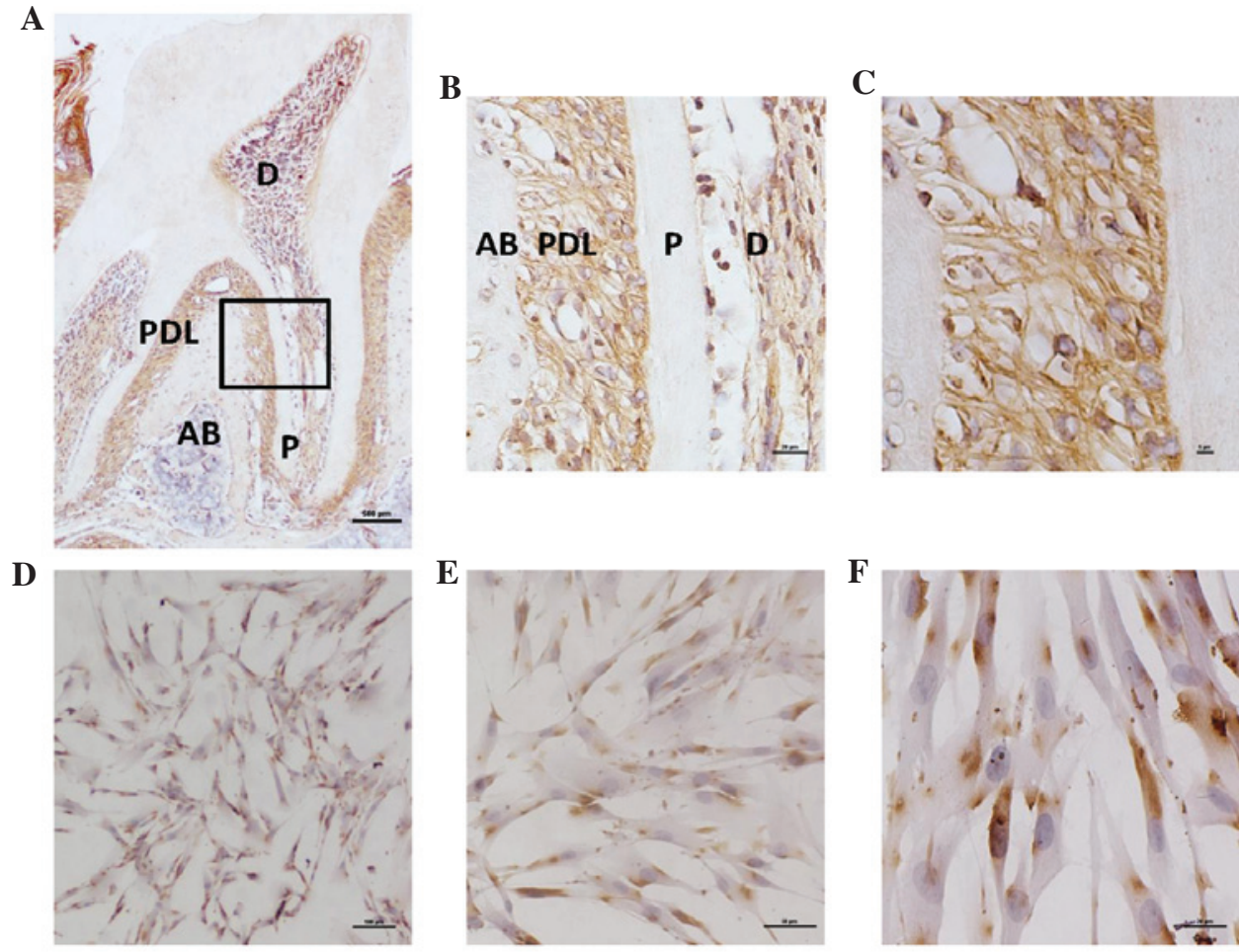

Figure 1. GGT in PDL tissues of mice and hPLCs. (A) Immunohistochemical analysis of GGT in PDL tissues of the lower second molars of four-week-old C57BL/6 mice (scale bar, $500 \mu \mathrm{m}$; diaminobenzidine and hematoxylin staining). (B) Magnified area within the black rectangle in A (magnification, x20; scale bar, $20 \mu \mathrm{m}$ ). (C) The PDL tissue area within the black rectangle in A (magnification, x40; scale bar, $5 \mu \mathrm{m}$ ). (D) Immunohistological localization of GGT in hPLCs at passage three (magnification, x4; scale bar, $100 \mu \mathrm{m}$ ). (E and F) Magnified areas from D (magnification, x10 and x20, respectively; scale bars, 50 and $20 \mu \mathrm{m}$, respectively. AB, alveolar bone; P, pulp; D, dentin; GGT, gamma-glutamyl transferase; hPLC, human periodontal ligament cell; PDL, periodontal ligament.

Wound healing assay. To test the effects of GGsTOP on cell migration, hPLCs $\left(5 \times 10^{5}\right.$ cells/well $)$ were seeded into six-well plates and cultured until $80-90 \%$ confluent for the in vitro scratch-wound assay (11). Briefly, a mark was made on the outside of each well before the experiment, so as to capture the same image area at each time point, using NIS-Elements Basic Research software. A uniform scratch wound was created by dragging a plastic pipette tip across the culture dish. The cells were incubated with medium containing $10 \mu \mathrm{M}$ GGsTOP or $10 \mu \mathrm{M}$ GGsTOP+3 mM NAC, while control cells did not receive any treatment. At 0,24 and $48 \mathrm{~h}$ after the wounding procedure, images were taken in each group under light microscopy (Eclipse 80i; Nikon). To calculate the number of cells migrated into the wound area, cells were fixed with $4 \%$ paraformaldehyde for $30 \mathrm{~min}$ and stained with DAPI at $48 \mathrm{~h}$, then were observed under a fluorescence microscope (Eclipse Ti-S; Nikon).

Western blot analysis. hPLCs $\left(1 \times 10^{6}\right.$ cells/well) were incubated in medium containing $10 \mu \mathrm{M}$ GGsTOP, $20 \mu \mathrm{M}$ GGsTOP or $10 \mu \mathrm{M}$ GGsTOP+3 mM NAC for $72 \mathrm{~h}$ in a six-well plate, while the control cells did not undergo any treatment. Cell protein was extracted using radioimmunoprecipitation assay buffer (Beyotime Institute of Biotechnology), and quantified using the BCA method (Beyotime Institute of Biotechnology). The protein extracts were resolved on a $10 \%$ tris-glycine acrylamide gel (Invitrogen; Thermo Fisher Scientific, Inc., Waltham, MA, USA) under denaturing conditions and then electrotransferred onto polyvinylidene difluoride membranes
(Immobilon-P; Millipore, Billerica, MA, USA). The membranes were serially incubated with specific primary antibodies: Rabbit anti- $\alpha$-smooth muscle actin (SMA; cat. no. ab5694; 1:1,000 dilution; Abcam) and mouse anti- $\beta$-actin (cat. no. sc-47778; 1:1,000; Santa Cruz Biotechnology, Inc., Dallas, TX, USA) overnight at $4^{\circ} \mathrm{C}$. The membranes were washed and incubated with the following secondary antibodies: Peroxidase-Conjugated AffiniPure Goat AntiRabbit IgG (cat. no. ZB-5301; 1:20,000; ZSGB-BIO) and Peroxidase-Conjugated AffiniPureGoat Anti-Mouse IgG (cat. no. ZB-5305; 1:20,000; ZSGB-BIO). Signals were visualized with an enhanced chemiluminescence kit (ECL Plus; GE Healthcare, Little Chalfont, UK). The gray density of protein was analyzed by the Chemiluminescence imaging system Smart ChemiTM420 (IV) (Beijing Sage Creation Science Co., Ltd., Beijing, China).

Immunofluorescence analysis. hPLCs $\left(5 \times 10^{5}\right.$ cells/well) were incubated with a medium containing $10 \mu \mathrm{M}$ GGsTOP, $20 \mu \mathrm{M}$ GGsTOP or $10 \mu \mathrm{M}$ GGsTOP $+3 \mathrm{mM}$ NAC for $72 \mathrm{~h}$ in a six-well plate. The control cells and negative control cells did not undergo any treatment. The cells were fixed with $4 \%$ paraformaldehyde for $30 \mathrm{~min}$ and blocked with $3 \%$ bovine serum albumin (Beyotime Institute of Biotechnology) for $1 \mathrm{~h}$ at room temperature. The samples were incubated with 1:500 diluted rabbit anti-human collagen-I polyclonal antibody (cat. no. ab34710; Abcam) overnight at $4^{\circ} \mathrm{C}$. Rabbit immunoglobulin (Ig)G (cat. no. sc-66928; 1:40 dilution; Santa Cruz Biotechnology, Inc.) was applied for the negative control. A fluorescein isothiocyanate 
(FITC)-conjugated goat polyclonal anti-rabbit IgG was used as the secondary antibody (1:160; cat. no. ZF-0314; ZSGB-BIO) for $1 \mathrm{~h}$ at room temperature. For nuclear detection, the samples were reacted with DAPI for 3 min. Immunofluorescence was observed under a fluorescence microscope (Nikon).

Statistical analysis. All values are expressed as the mean \pm standard deviation. Statistical analysis was performed by one-way analysis of variance followed by Dunnett's test. The processing of the data was performed using SPSS version 19.0.1 for Windows (SPSS Inc., USA). P<0.05 was considered to indicate a statistically significant difference.

\section{Results}

Localization of GGT in PDL tissues from mice and hPLCs. Immunohistochemical analysis revealed the presence of GGT-positive cells throughout the mandibular tissues, including the PDL and pulp. The amount of GGT in PDL tissues was greater than that in the pulp, whereas no staining was observed in the alveolar bone (Fig. 1A-C). Positive staining was distinct in the plasma membrane but not in the nucleus of the cells (Fig. 1D-F).

GGsTOP inhibits GGT activity in hPLCs. To explore possible mechanisms underlying the effects of GGT inhibition on PDL tissue, the effect of GGsTOP on GGT activity in hPLCs was evaluated. A biochemical assay of the homogenates showed that the GGT activity in hPLCs was $2.93 \pm 0.7 \mathrm{U} / \mathrm{g}$ protein, while that in HepG2 cells was $23.05 \pm 1.5 \mathrm{U} / \mathrm{g}$ protein (Table I). A significant decrease in GGT activity was noted in the hPLCs within $2 \mathrm{~h}$ of treatment with GGsTOP $(10 \mu \mathrm{M})$.

GGsTOP does not affect the viability of hPLCs. Light microscopic examination indicated that GGsTOP treatment did not produce any cytotoxic effects in hPLCs relative to cells in the untreated group (Fig. 2A). The hPLCs appeared to be elongated, without any interspersed non-viable cells. Cell survival was further assessed using the MTT method, which revealed that cell viability was not significantly affected by GGsTOP treatment $(5,10,20$ and $50 \mu \mathrm{M}$ for 1, 3 and 5 days) (Fig. 2B). It was noted that treatment with $10 \mu \mathrm{M}$ GGsTOP resulted in enhanced proliferation of hPLCs when compared with the control cells after 7 days $(\mathrm{P}<0.05)$. These results suggested that application of GGsTOP in the 5-50 $\mu \mathrm{M}$ range was safe for hPLCs under the conditions used in the present study.

GGSTOP induces the generation of ROS in hPLCs. Intracellular ROS were measured by pre-incubating the cells with DCF-DA to monitor the ROS-sensitive fluorescent product of DCFH (Fig. 3). Incubation with GGsTOP $(10 \mu \mathrm{M})$ resulted in elevated levels of ROS at 120 min of incubation, as demonstrated by fluorescence microscopy (Fig. 3A).

Quantitative analysis of fluorescence microscopy images at various time-points allowed for assessment of the time-dependency of ROS induction by GGsTOP in hPLCs (Fig. 3B). hPLCs exposed to GGsTOP were found to exhibit a transient increase in intracellular ROS over time; intracellular ROS were significantly elevated at $15 \mathrm{~min}$ of stimulation, peaked at $120 \mathrm{~min}$ and declined at $180 \mathrm{~min}$. However, co-treatment
Table I. GGT activity (U/g protein) in different groups.

\begin{tabular}{lr}
\hline Group & GGT activity \\
\hline hPLCs & $2.93 \pm 0.70$ \\
GGsTOP+hPLCs & $0.76 \pm 0.40$ \\
HepG2 & $23.05 \pm 1.50$
\end{tabular}

Values are expressed as the mean \pm standard deviation $(n=6)$. GGT, gamma-glutamyl transferase; hPLC, human periodontal ligament cell.
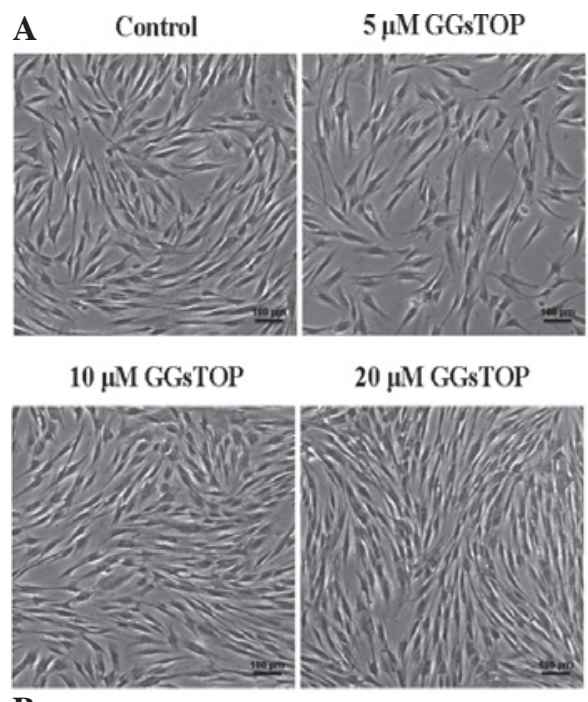

$50 \mu \mathrm{M}$ GGsTOP

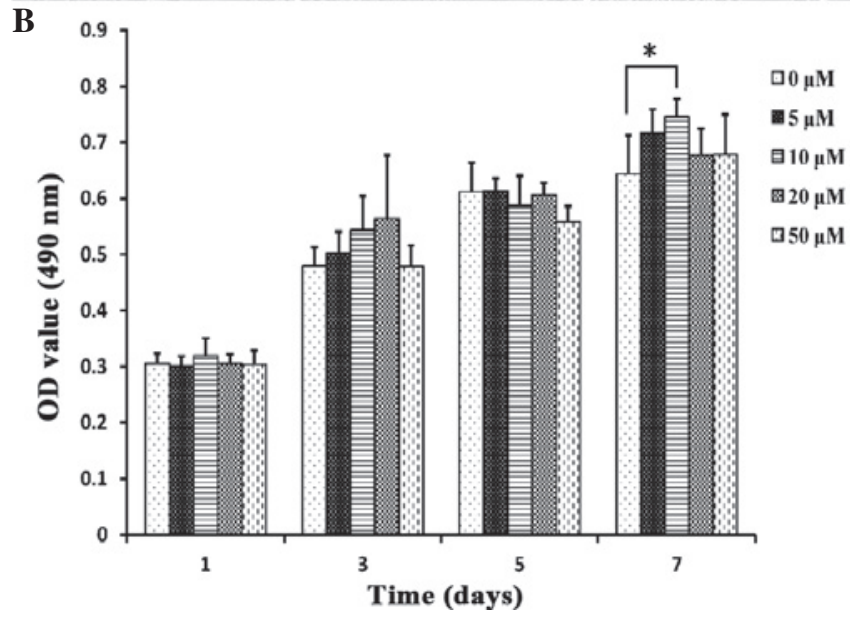

Figure 2. Cellular viability and shape of hPLCs maintained with GGsTOP. (A) Microscopy images of hPLCs three days after treatment with various concentrations of GGsTOP. Cells without any treatment were used as controls (scale bar, $100 \mu \mathrm{m}$ ). (B) Bar graphs depicting the time-dependent increase in hPLC proliferation after GGsTOP treatment. Values are expressed as the mean \pm standard deviation. ${ }^{*} \mathrm{P}<0.05$. hPLC, human periodontal ligament cell; OD, optical density.

with NAC completely inhibited the GGsTOP-induced ROS production.

GGSTOP is associated with altered migration of hPLCs via the ROS pathway. To further determine whether GGsTOP treatment has a beneficial effect on the migration of cultured hPLCs, A scratch-wound assay was performed as described by Gao et al (11). GGsTOP was found to facilitate the migra- 
A

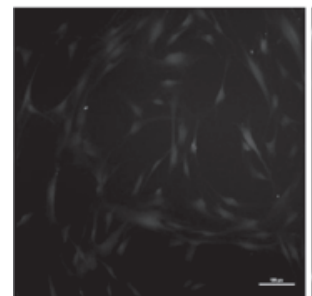

Control

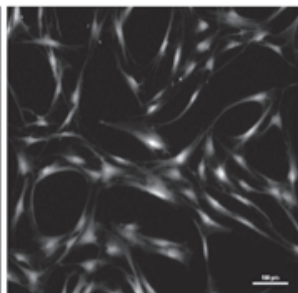

GGsTOP

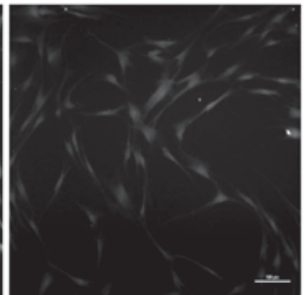

GGsTOP+NAC
B

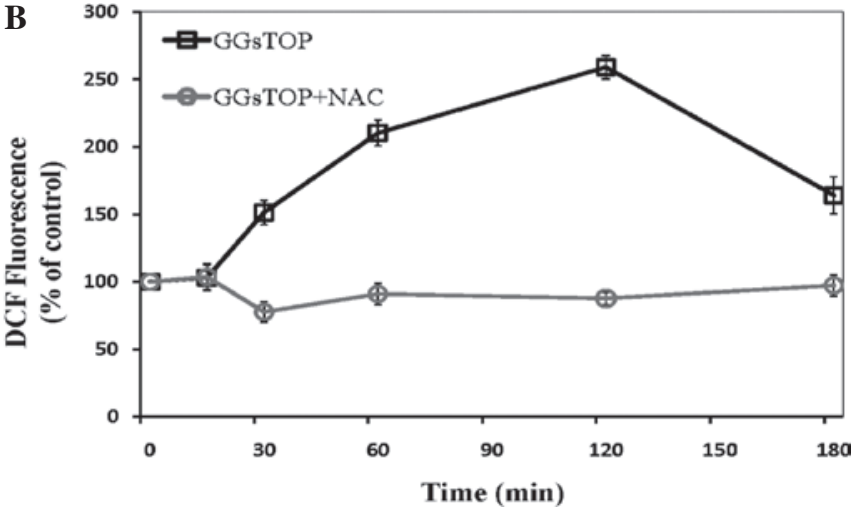

Figure 3. GGsTOP induces ROS generation in hPLCs. (A) hPLCs were pre-incubated with DCFH-DA (10 mM) to detect ROS-induced fluorescence Unstimulated (control), GGsTOP $(10 \mu \mathrm{M})$-stimulated, and NAC (10 mM)+GGsTOP (10 $\mu \mathrm{M})$-stimulated cells observed at $120 \mathrm{~min}$ (scale bar, $100 \mu \mathrm{m})$. (B) Graph depicting the time course of ROS generation in cells stimulated with GGsTOP at 0,15,30,60,120 and 180 min, with (O) or without (प) NAC. DCF fluorescence was expressed as the percentage of the control. Values are expressed as the mean \pm standard deviation ( $n=3$ ). ROS, reactive oxygen species; NAC, $N$-acetylcysteine; DCFH-DA, dichlorodihydrofluorescein diacetate; hPLC, human periodontal ligament cell.

A

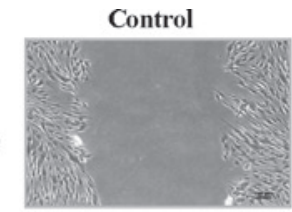

Time (0 h)

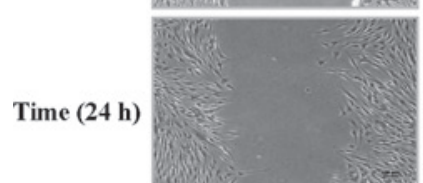

Time $(48 \mathrm{~h})$ (1)

DAPI

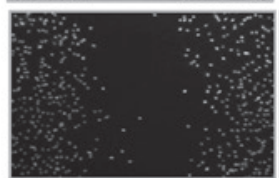

GGsTOP
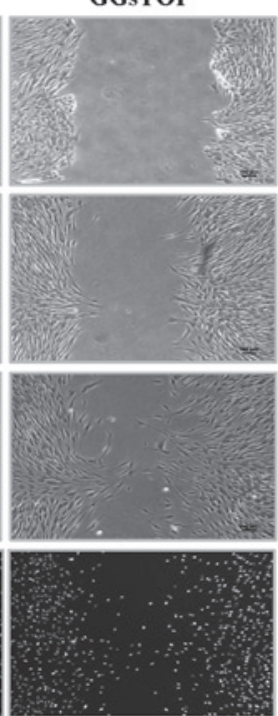

GGsTOP+NAC

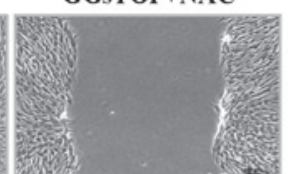

B

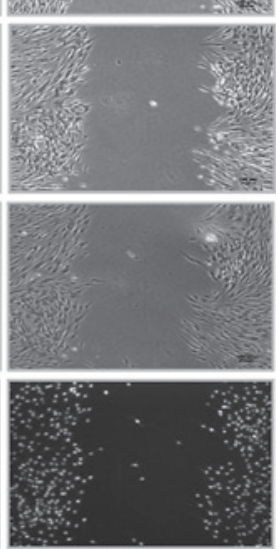

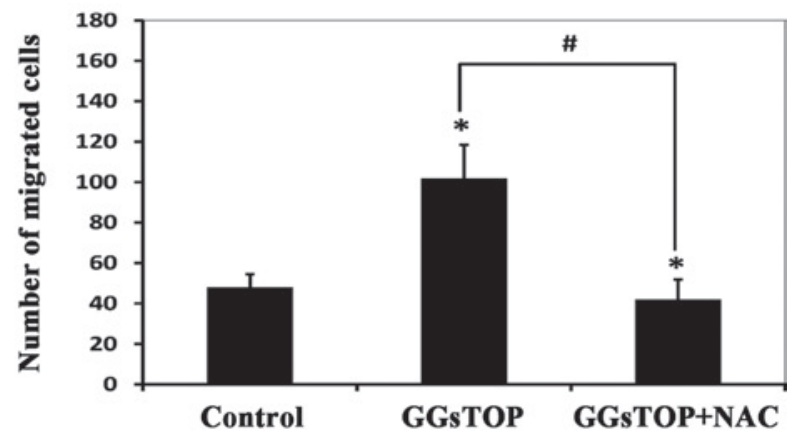

Figure 4. Effect of ROS on GGsTOP-induced cell migration and wound closure in hPLCs. (A) Uniform scrape wounds to the monocell layer were generated in the following groups: Unstimulated cells (control), cells stimulated with $10 \mu \mathrm{M}$ GGsTOP or cells stimulated with $10 \mu \mathrm{M}$ GGsTOP in NAC ( 3 mM)-containing medium (GGsTOP+NAC). (A) Images of wound closure and cell migration were captured at 24 and $48 \mathrm{~h}$ under a microscope (scale bar, $100 \mu \mathrm{m}$ ). DAPI staining was performed to assess cell migration throughout the scrape wound at $48 \mathrm{~h}$. (B) Quantitative analysis of the migratory cell numbers crossing the wound edge at $48 \mathrm{~h}$ following wounding. Values are expressed as the mean \pm standard deviation. The above experiment was repeated three times. "P<0.05 vs. control; ${ }^{\#} \mathrm{P}<0.05$ as indicated. NAC, $N$-acetylcysteine; hPLC, human periodontal ligament cell.

tion of cells when compared with the control cells (Fig. 4A). Migration of the control cells was slow at $24 \mathrm{~h}$ and was not much different at $48 \mathrm{~h}$ post-wounding. However, increased cell migration was observed in the GGsTOP group, in which the wounded area was reduced by $\sim 50 \%$ at $48 \mathrm{~h}$ (Fig. 4B). By contrast, in the presence of NAC, the wound closure stimulated by GGsTOP was completely inhibited at 24 and $48 \mathrm{~h}$ post-wounding.

GGSTOP increases COL-I and $\alpha$-SMA synthesis in hPLCs. The expression of $\alpha$-SMA protein in hPLCs stimulated by GGsTOP was analyzed by western blot analysis (Fig. 5A). Notably, at $55 \mathrm{kDa}$ an extra band was observed, representing vimentin with which the antibody had cross-reacted. Vimentin is predominantly expressed in mesenchymal cells. The results showed significantly elevated levels of $\alpha$-SMA in the cells treated with 10 or $20 \mu \mathrm{M}$ GGsTOP for $72 \mathrm{~h}$ when compared with the control $(\mathrm{P}<0.01)$, while this effect was completely inhibited by co-treatment with NAC (Fig. 5A). Immunofluorescent staining revealed low levels of COL-I in the hPLCs under normal conditions (Fig. 5B). By contrast, cells stimulated with 10 or $20 \mu \mathrm{M}$ GGsTOP contained elevated levels of COL-I, while co-incubation with the anti-oxidant NAC completely inhibited these increases. Staining with a control $\mathrm{IgG}$ used as the primary antibody did not reveal any positive reaction. It is well known that NAC is an antioxidant which reduces intracellular ROS production. Our results (Fig. 3) also demonstrated that GGsTOP-stimulated ROS was totally eliminated when NAC was present in the cells. These findings suggested that treatment with GGsTOP significantly increased COL-I and $\alpha$-SMA in hPLCs, and that the generation of ROS was essential in this process. 

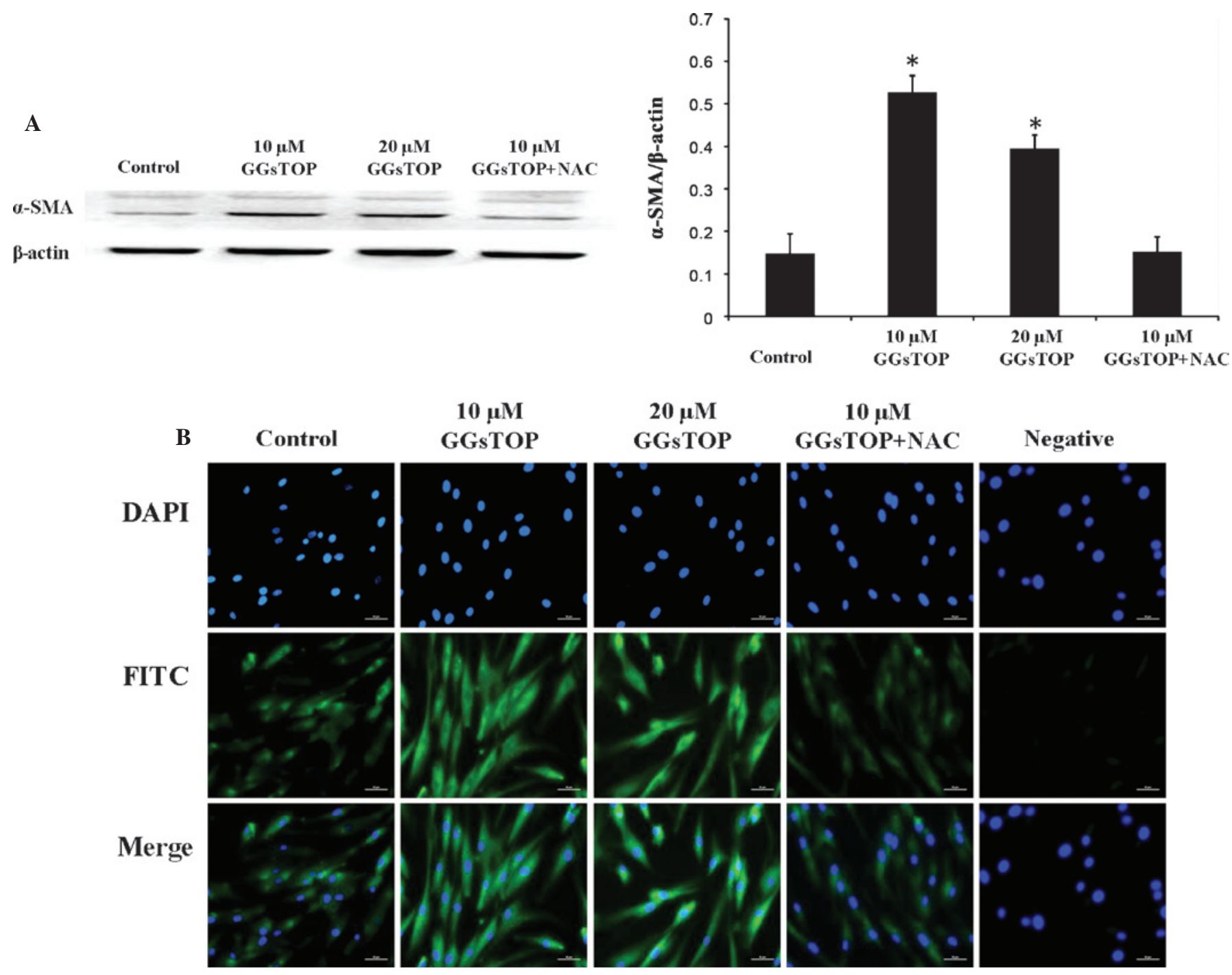

Figure 5. Expression of COL-I and $\alpha$-SMA in GGsTOP-treated hPLCs. (A) GGsTOP increased $\alpha$-SMA protein levels in hPLCs. Western blots revealed clear bands at $42 \mathrm{kDa}$, representing $\alpha$-SMA. Additional bands representing vimentin, with which the antibody had cross-reacted, were observed at $55 \mathrm{kDa}$. Quantitative representation of the western blots in A. Values are expressed as the mean \pm standard deviation $\left(\mathrm{n}=3 ;{ }^{*} \mathrm{P}<0.05\right.$ vs. control). (B) Immunofluorescent analysis of COL-I (FITC) in GGsTOP (10,20 $\mu \mathrm{M}$ )-treated hPLCs cultured with or without NAC (3 mM). After three days of culture, the hPLCs were incubated with a control mouse immunoglobulin G (Negative group) or an anti-COL-I antibody (green). Nuclei were stained with DAPI (blue). Scale bars, $50 \mu \mathrm{m}$. COL, collagen; SMA, smooth muscle actin; FITC, fluorescein isothiocyanate; hPLC, human periodontal ligament cell; NAC, $N$-acetylcysteine.

\section{Discussion}

GGT is an important enzyme required for the maintenance of steady-state glutathione concentration inside as well as outside of the cells (extracellular fluids), and is used as a diagnostic marker for liver disease in the clinic. However, to the best of our knowledge, the localization and functions of GGT in intact PDL tissues have not been previously reported. Levels of GGT vary considerably among normal tissues and organs. Strong immunoreactivity for this enzyme has been reported in the renal proximal convoluted tubule, hepatic bile canaliculi and brain capillaries (12). The present study was the first to report the presence of GGT-positive cells in mandibular tissues, including PDL and pulp tissues. The activity of GGT was found to be eight times greater in HepG2 cells compared with that in hPLCs, indicating a great variability among the two cell types. In addition, it was demonstrated that GGsTOP markedly inhibited GGT activity in hPLCs after $2 \mathrm{~h}$. The present study further investigated whether intervention of GGT activity had any effect on PDL cells under physiological conditions.

In the present study, an MTT assay demonstrated that the viability of hPLCs was not affected by GGsTOP, indicating its suitability for the treatment of these cells. Furthermore, microscopic observation revealed that hPLCs appeared elongated, without the presence of any interspersed non-viable cells. This morphology conformed to that of a healthy fibroblastic phenotype (13). GGT inhibition by acivicin has been shown to arrest cell growth (14). However, a previous study has reported that acivicin increases the lifespan, and may therefore be applicable for the development of anti-aging therapies (15). It is therefore likely that the effects of GGsTOP on proliferation may depend on the cell type, stage of differentiation and culture conditions.

The observation of the present study that GGsTOP induced a transient elevation of intracellular ROS in hPLCs agrees with the results reported by Zalata et al (16), according to which GGT activity was inversely correlated with ROS production in the spermatozoa, an observation also consistent with that 
of Johnsen et al (17). These results also indicated that hPLCs, analogous to other non-phagocytic cells, can produce intracellular ROS during stimulation (18).

ROS are thought to act as signaling molecules that direct the changes necessary for cell movement. To clarify whether GGsTOP promotes cell migration in cultured hPLCs via ROS, the antioxidant NAC was added to the medium to eradicate intracellular ROS generation. The results indicated that GGsTOP facilitates cell migration at $48 \mathrm{~h}$. However, the function of GGsTOP was blocked when NAC was also present in the medium. To determine whether GGsTOP promotes cell migration in cultured hPLCs, a scratch-wound test was performed. The results indicated that GGsTOP facilitates cell migration, which may be attributed to the rise in intracellular ROS levels. However, the underlying mechanisms involved in these processes remain elusive. Previous studies suggested that the ROS that form in the superficial cell layers next to the wound interface are susceptible to mechanical damage, which can directly lead to the activation of the mitogen-activated protein kinase signaling pathway. Evidence also suggested that ROS-mediated enhancement of fibroblast migration is associated with the bone morphogenetic protein/SMAD signaling pathway (19). The level of ROS is a key factor in the wound healing process. Therefore, it requires a well-orchestrated and dynamic response to maintain the cellular redox homeostasis, which is achieved by various oxidordeuctases and small molecules. GSH is of great importance as it serves as a cellular redox buffer. A role for GSH in wound repair has been suggested, since the GSH level was reduced in skin wounds, and topical treatments in diabetic mice with GSH accelerated the repair processes (20). GGT, as a rate-limiting enzyme, can influence intracellular de novo glutathione synthesis. Thus, GGT may be crucial for efficient wound healing. However, the exact mechanism by which activation of cell surface receptors activates GGsTOP and generates ROS during cell migration remains elusive. In addition, the identities of the redox-regulated proteins that are oxidized remain unknown. However, GGT activity is known to mediate nuclear factor-kappa B activation, which can modulate their function and influence cell migration (21).

In the present study, the effects of GGT activity in hPLCs under physiological conditions were assessed, as it has been reported that numerous growth factors and chemoattractants regulate tissue regeneration, accompanied by the production of intracellular ROS in various cell types. Transforming growth factor $\beta 1$ (TGF $\beta 1$ ) can stimulate ROS in fibroblasts, while platelet-derived growth factor (PDGF) induces ROS production in lens epithelial cells and ROS are also essential factors associated with epidermal growth factor (EGF)-stimulated corneal epithelial cell migration $(4,18,22)$. Choe et al (23) demonstrated that ROS produced by glucose oxidase increased the viability, proliferation and osteoblastic differentiation of hPLCs. In addition, it is known that inhibition of GGT activity at the surface of human myeloid cells correlates with macrophage maturation and transforming growth factor- $\beta$ production in several cell types (24). TGF- $\beta 1$ is considered to be a key regulator of COL I and $\alpha$-SMA expression, as well as extracellular matrix components in hPLCs (25). Collagen fibers have a pivotal role in anchoring the tooth to the alveolar bone socket and are primarily composed of type I collagen (26). Expression of $\alpha$-SMA is generally associated with high remodeling capacity as seen in the periodontal ligament during conditions of increased remodeling (27). For these reasons, the present study analyzed the effects of GGsTOP on the protein levels of COL-I and $\alpha$-SMA. The results indicated that inhibition of GGT activity using various concentrations of GGsTOP obviously increased the expression of COL-I and $\alpha$-SMA (Fig. 5). These findings suggested that GGsTOP induces the differentiation of immature hPLCs into functional PDL fibroblasts through the upregulation of COL-I and $\alpha$-SMA. However, the underlying mechanisms warrant further evaluation.

In conclusion, the present study demonstrated for the first time, to the best of our knowledge, that GGT is present in PDL tissues. Treatment of hPLCs with GGT inhibitor GGsTOP increased cell migration and ROS levels, and enhanced the expression levels of COL-I and $\alpha$-SMA, which have vital roles during wound healing. Of note, the anti-oxidant NAC inhibited these effects of GGsTOP, possibly through inhibition of ROS. In the light of the absence of any cytotoxic effects of GGsTOP, these results indicated that GGsTOP may be used for the treatment of periodontal ligament wound healing.

\section{Acknowledgements}

The present study was supported by the National Natural Science Foundation of China (grant no. 81170960) and the Special Foundation for Sino-Russian Translational Medicine Research Center of Harbin Medical University (grant no. CR201412).

\section{References}

1. Nomura Y, Ishikawa M, Yashiro Y, Sanggarnjanavanich S, Yamaguchi T, Arai C, Noda K, Takano Y, Nakamura Y and Hanada N: Human periodontal ligament fibroblasts are the optimal cell source for induced pluripotent stem cells. Histochem Cell Biol 137: 719-732, 2012.

2. Cáceres M, Martínez C, Martínez J and Smith PC: Effects of platelet-rich and -poor plasma on the reparative response of gingival fibroblasts. Clin Oral Implants Res 23: 1104-1111, 2012.

3. Fu XJ, Peng YB, Hu YP, Shi YZ, Yao M and Zhang X: NADPH oxidase 1 and its derived reactive oxygen species mediated tissue injury and repair. Oxid Med Cell Longev 2014: 282854, 2014.

4. Chen KC, Zhou Y, Xing K, Krysan K and Lou MF: Platelet derived growth factor (PDGF)-induced reactive oxygen species in the lens epithelial cells: The redox signaling. Exp Eye Res 78: 1057-1067, 2004.

5. Lu SC: Regulation of hepatic glutathione synthesis: Current concepts and controversies. FASEB J 13: 1169-1183, 1999.

6. Wickham S, WestMB,CookPFand Hanigan MH: Gamma-glutamyl compounds: Substrate specificity of gamma-glutamyl transpeptidase enzymes. Anal Biochem 414: 208-214, 2011.

7. Rojas E, Valverde M, Kala SV, Kala G and Lieberman MW: Accumulation of DNA damage in the organs of mice deficient in gamma-glutamyltranspeptidase. Mutat Res 447: 305-316, 2000.

8. Yamamoto S, Watanabe B, Hiratake J, Tanaka R, Ohkita M and Matsumura Y: Preventive effect of GGs Top, a novel and selective $\gamma$-glutamyl transpeptidase inhibitor, on ischemia/reperfusion-induced renal injury in rats. J Pharmacol Exp Ther 339: 945-951, 2011.

9. Huseby NE and Strömme JH: Practical points regarding routine determination of gamma-glutamyl transferase (gamma-GT) in serum with a kinetic method at 37 degrees C. Scand J Clin Lab Invest 34: 357-363, 1974.

10. Cannito $S$, Novo $E$, Compagnone $A$, Valfrè di Bonzo L, Busletta $C$, Zamara E, Paternostro C, Povero D, Bandino A, Bozzo F, et al: Redox mechanisms switch on hypoxia-dependent epithelial-mesenchymal transition in cancer cells. Carcinogenesis 29: 2267-2278, 2008 . 
11. Gao C, Negash S, Guo HT, Ledee D, Wang HS and Zelenka P: CDK5 regulates cell adhesion and migration in corneal epithelial cells. Mol Cancer Res 1: 12-24, 2002.

12. Hanigan $\mathrm{MH}$ and Frierson HF Jr: Immunohistochemical detection of gamma-glutamyl transpeptidase in normal human tissue. J Histochem Cytochem 44: 1101-1108, 1996.

13. Eckes B, Zweers MC, Zhang ZG, et al: Mechanical tension and integrin $\alpha 2 \beta 1$ regulate fibroblast functions. J Invest Dermatol 11: 66-72, 2006.

14. del Bello B, Paolicchi A, Comporti M, Pompella A and Maellaro E: Hydrogen peroxide produced during gamma-glutamyl transpeptidase activity is involved in prevention of apoptosis and maintainance of proliferation in U937 cells. FASEB J 13: 69-79, 1999.

15. Stephan J, Franke J and Ehrenhofer-Murray AE: Chemical genetic screen in fission yeast reveals roles for vacuolar acidification, mitochondrial fission, and cellular GMP levels in lifespan extension. Aging Cell 12: 574-583, 2013.

16. Zalata A, Hafez T, Mahmoud A and Comhaire F: Relationship between resazurin reduction test, reactive oxygen species generation, and gamma-glutamyltransferase. Hum Reprod 10: 1136-1140, 1995.

17. Johnsen O, Eliasson R and Samuelson U: Conditioning effect of seminal plasma on the lipid peroxide potential of washed human spermatozoa. Acta Physiol Scand 116: 305-307, 1982.

18. Liu RM, Liu Y, Forman HJ, Olman M and Tarpey MM Glutathione regulates transforming growth factor-beta-stimulated collagen production in fibroblasts. Am J Physiol Lung Cell Mol Physiol 286: L121-L128, 2004.

19. Tandon N, Cimetta E, Villasante A, Kupferstein N, Southall MD Fassih A, Xie J, Sun Y and Vunjak-Novakovic G: Galvanic microparticles increase migration of human dermal fibroblasts in a wound-healing model via reactive oxygen species pathway. Exp Cell Res 320: 79-91, 2014.
20. Mudge BP, Harris C, Gilmont RR, Adamson BS and Rees RS: Role of glutathione redox dysfunction in diabetic wounds. Wound Repair Regen 10: 52-58, 2002.

21. Djavaheri-Mergny $M$, Accaoui MJ, Rouillard D and Wietzerbin J: Gamma-glutamyl transpeptidase activity mediates NF-kappaB activation through lipid peroxidation in human leukemia U937 cells. Mol Cell Biochem 232: 103-111, 2002.

22. Svegliati S, Cancello R, Sambo P, Luchetti M, Paroncini P, Orlandini G, Discepoli G, Paterno R, Santillo M, Cuozzo C, et al: Platelet-derived growth factor and reactive oxygen species (ROS) regulate Ras protein levels in primary human fibroblasts via ERK1/2. Amplification of ROS and Ras in systemic sclerosis fibroblasts. J Biol Chem 280: 36474-36482, 2005.

23. Choe Y, Yu JY, Son YO, Park SM, Kim JG, Shi X and Lee JC: Continuously generated $\mathrm{H}_{2} \mathrm{O}_{2}$ stimulates the proliferation and osteoblastic differentiation of human periodontal ligament fibroblasts. J Cell Biochem 113: 1426-1436, 2012.

24. Bauvois B, Laouar A, Rouillard D and Wietzerbin J: Inhibition of gamma-glutamyl transpeptidase activity at the surface of human myeloid cells is correlated with macrophage maturation and transforming growth factor beta production. Cell Growth Differ 6: 1163-1170, 1995.

25. Fujita T, Shiba H, Van Dyke TE and Kurihara H: Differential effects of growth factors and cytokines on the synthesis of SPARC, DNA, fibronectin and alkaline phosphatase activity in human periodontal ligament cells. Cell Biol Int 28: 281-286, 2004.

26. Narayanan AS and Page RC: Connective tissues of the periodontium: A summary of current work. Coll Relat Res 3: 33-64, 1983.

27. Tomasek JJ, Gabbiani G, Hinz B, Chaponnier C and Brown RA: Myofibroblasts and mechano-regulation of connective tissue remodelling. Nat Rev Mol Cell Biol 3: 349-363, 2002. 